\title{
ASPECTS OF MAGNETISM IN HEAVY-ELECTRON METALS
}

\author{
H.R. OTT \\ Laboratorium für Festkörperphysik, ETH-Hönggerberg, 8093 Zürich, Switzerland
}

For some time it was believed that heavy-mass electrons at low temperatures, in special rare-earth and actinide compounds, were due to a particular suppression of magnetic order in these materials. Various experiments reveal, however, that magnetic order may occur from a heavy-electron state and that also a heavy-electron state may develop in the presence of a magnetically-ordered matrix. Even in materials with no sign of a cooperative phase transition, microscopic experimental probes give evidence for strong magnetic correlations, involving only very tiny $\left(<10^{-1} \mu_{\mathrm{B}}\right)$ ordered moments, however. All these experimental observations indicate that the heavy-electron state cannot always be regarded as a simple Fermi liquid in the usual sense. Various interactions of similar strength need to be considered. It is suggested that the balance of these interactions may lead to electronic inhomogeneities in $k$ space. Both in magnetically-ordered or superconducting systems, unexpected electronic degrees of freedom appear to exist. In other cases experimental observations suggest magnetic inhomogeneities in real space which are not simply due to chemical inhomogeneities. Different examples of these various possibilities are demonstrated and discussed.

PACS numbers: 75.20.-g, 75.20.Hr, 75.30.-m, 75.30.Mb

\section{Introduction}

The questions regarding the stability of magnetic moments in metals have been pursued for a long time but the ideas considered in the early works of Friedel [1], Blandin [2], Anderson [3] and Kondo [4] still provide the essential background for discussing recent and new experimental observations. Simple metals are believed to be well understood with a Fermi-liquid-type behaviour at low temperatures and, quite often, a transition to a superconducting state because of electron-phonon interaction. Less transparent is the behaviour of $d$ - and $f$-electron transition metals and compounds. The most recent interest in such materials is connected with a class of substances that was termed heavy-electron systems. Here the electron-electron interactions dominate the electronic properties and therefore, many-body effects can no longer be neglected or treated with simple approximation schemes. 
Materials under consideration here contain ions with configurationally well defined magnetic moments, due to incompletely-filled atomic electron orbitals, on regular lattice sites. These moments necessarily interact with the ensemble of conduction electrons and it may be expected that the adopted ground state is of some magnetically-ordered variety. In this case it is usually assumed that the Ruderman-Kittel-Kasuya-Yoshida (RKKY) interaction via conduction electrons $[5,6]$ couples the individual ionic moments. The above-mentioned theoretical work essentially considered the stability of an isolated single magnetic moment in a metallic environment. The main obstacle with the new developments is to treat the analogous problem for arrays of such moments in 3 dimensions. Obviously, there are competing trends in the magnetic moment-conduction electron interactions, either favouring a cooperative alignment of moments or else, leading to a reduction or even full compensation of moments on a single-ion level, whereby also cooperatively compensated states ought to be considered [7].

Below we intend to discuss a few experimental observations which indicate that due to competing interactions of likely similar magnitude, ground states that are more complex than those envisaged before may be adopted. The main purpose of this discussion is to provide evidence for new aspects of inhomogeneities in these materials, namely a probably not structurally-related anisotropy of electronic states in $k$ space and a new type of inhomogeneous magnetic correlations in real space. These selected examples may serve as a guideline for future explorations in this field, both in experinent and hopefully also in theory.

\section{Examples of experimental observations}

\section{1. $\mathrm{CeAl}_{3}$}

This compound has been for a long time considered to be a standard example where well defined local magnetic moments at low temperatures donate their degrees of freedom to a new kind of state with the characteristics of a Fermi-liquid state with strongly renormalized parameters, i.e., formed by quasiparticles with considerably enhanced effective masses. This view was based on the results of experiments probing thermal- and transport properties at high and low temperatures, i.e., between 0.05 and $300 \mathrm{~K}[8,9]$.

It was therefore very surprising when a $\mu$ SR experiment probing microscopically the magnetic properties of this material revealed a more complicated situation below temperatures of about $2 \mathrm{~K}[10]$. At very low temperatures and in zero magnetic field an oscillatory component in the $\mu \mathrm{SR}$ spectra indicated that at least quasistatic magnetic correlations inducing a corresponding magnetic field at the muon sites develop. The oscillation frequency decreases by approximately $15 \%$ between 0.05 and $0.7 \mathrm{~K}$ (see Fig. 1), and above this temperature the oscillatory component has vanished. The single frequency proves that the muons experiencing this field are trapped and decay on magnetically equivalent sites. The temperature dependence of the $\mu^{+}$-decay asymmetry indicates that the number of trapping sites exposed to the quasistatic field with a non-zero average value increases with decreasing temperature. As $T$ approaches $0 \mathrm{~K}$, virtually the whole sample volume is magnetically correlated [11]. The growth of the correlated regions occurs with- 


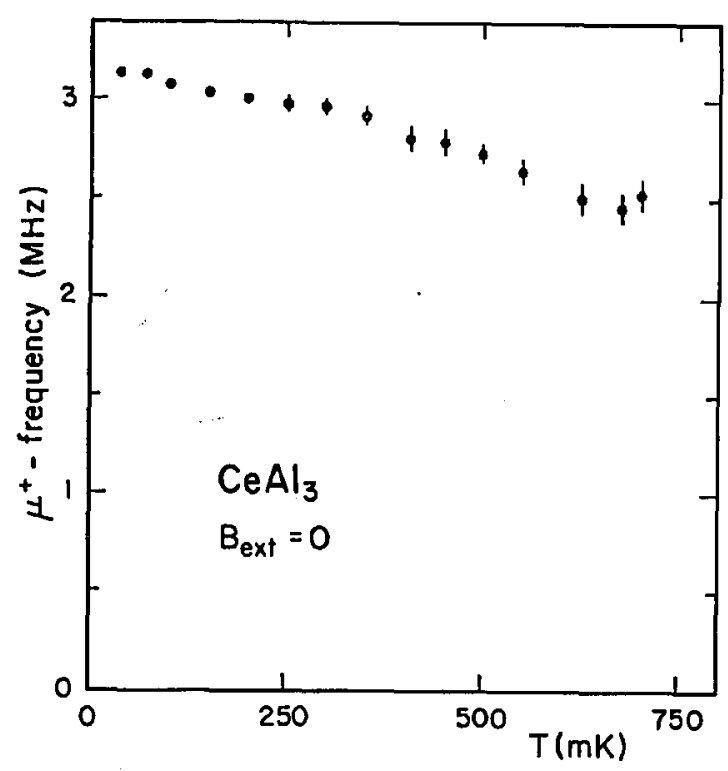

Fig. 1. Temperature dependence of the spontaneous $\mu^{+}$precession frequency in zero external magnetic field in $\overline{\mathrm{CeAl}}_{3}$ below $0.7 \mathrm{~K}$.

out any manifestation of a cooperative phase transition, as concluded before from experiments probing macroscopic properties.

Additional $\mu \mathrm{SR}$ experiments in non-zero external fields were used to identify the $\mu^{+}$-decay site in the crystal lattice with some reliability. Corresponding calculations and a thorough analysis of all available data indicated a value for $\mathrm{Ce}^{3+}$ derived moments of about $0.05 \mu_{\mathrm{B}} / \mathrm{Ce}$ below $0.7 \mathrm{~K}$ [12]. It is obvious that both this small value of the moments involved in the correlations and also the unusual, spatially inhomogeneous increase in magnetically-correlated volume with decreasing temperature need more attention.

More recent low-field NMR experiments on the same material and at temperatures between 0.035 and $20 \mathrm{~K}$ confirm to a large extent the previous microscopic observations [13]. Above $1.5 \mathrm{~K}$, fairly sharp and quadrupole-split resonance lines of ${ }^{27} \mathrm{Al}$ nuclei indicate a normal paramagnetic environment. Below $1.5 \mathrm{~K}$, however, a broad background intensity increases with decreasing temperature and at the lowest attained temperature of $0.036 \mathrm{~K}$, the NMR response has transformed into a broad peak with a width of a few hundred gauss (see Fig. 2). These spectra may again be taken as evidence for a growing portion of volume with a non-vanishing internal field of a magnitude that does not vary considerably with temperature.

Subsequent measurements of the spin-lattice relaxation rate $T_{1}^{-1}$ reveal a Korringa-type behaviour in this temperature interval, i.e., again no evidence for a cooperative phase transition. As expected, the Korringa constant is considerably enhanced if compared with the value obtained for pure $\mathrm{Al}$ [14]. 


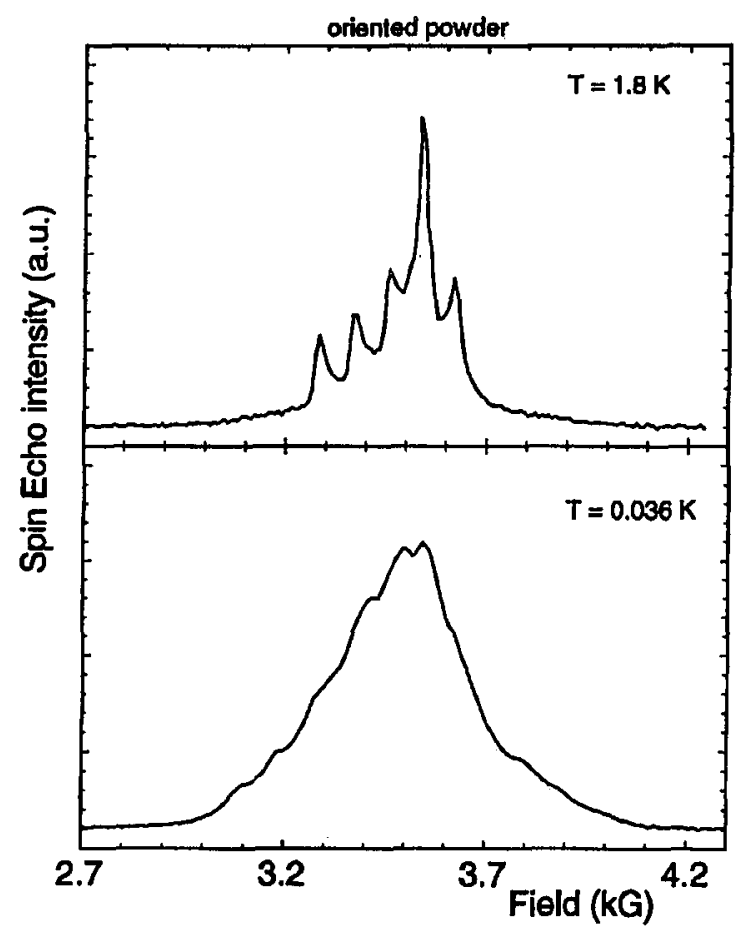

Fig. 2. NMR spectra of ${ }^{27} \mathrm{Al}$ in $\mathrm{CeAl}_{3}$ at a frequency of $3.964 \mathrm{MHz}$ and at low temperatures.

\section{2. $Y b P t B i$}

This compound is the end member of a series $R P t B i$, where $R$ stands for rare-earth elements. The crystal structure of these compounds is of cubic symmetry. Considering the unit-cell cube, all sites in the middle of the cube edges are unoccupied and also the potential site in the center of the cube is empty. The compounds with light rare-earth elements are semiconductors and only the $\mathrm{Yb}$ compound is a good metal with a residual resistance ratio (RRR) of the order of 20 [15]. Thus this compound may be regarded as being close to a metal-insulator transition. Recent Hall-effect data confirm this view by revealing a very small number of charge carriers per unit cell [16].

The most outstanding property of this substance is an extremely large specific heat at very low temperatures [17]. In Fig. 3 we show $C_{p}(T)$ between 0.1 and $1 \mathrm{~K}$. Between 0.5 and $1 \mathrm{~K}$, the specific heat is almost constant. Just below $0.4 \mathrm{~K}$, a small but distinct peak indicates a phase transition and below $0.35 \mathrm{~K}$, $C_{p}(T)$ varies almost linearly with temperature. Here, the ratio $C_{p}(T)$ is extremely large $\left(\approx 9 \mathrm{~J} /\left(\right.\right.$ mole $\left.^{2}\right)$ and the entropy related with this specific heat is $R \ln 2$ between 0 and $1 \mathrm{~K}$. Additional information concerning the phase transition just below $0.4 \mathrm{~K}$ has been obtained from $\chi(T)$ measurements [18]. A plot of $\chi_{\mathrm{ac}}(T)$ 


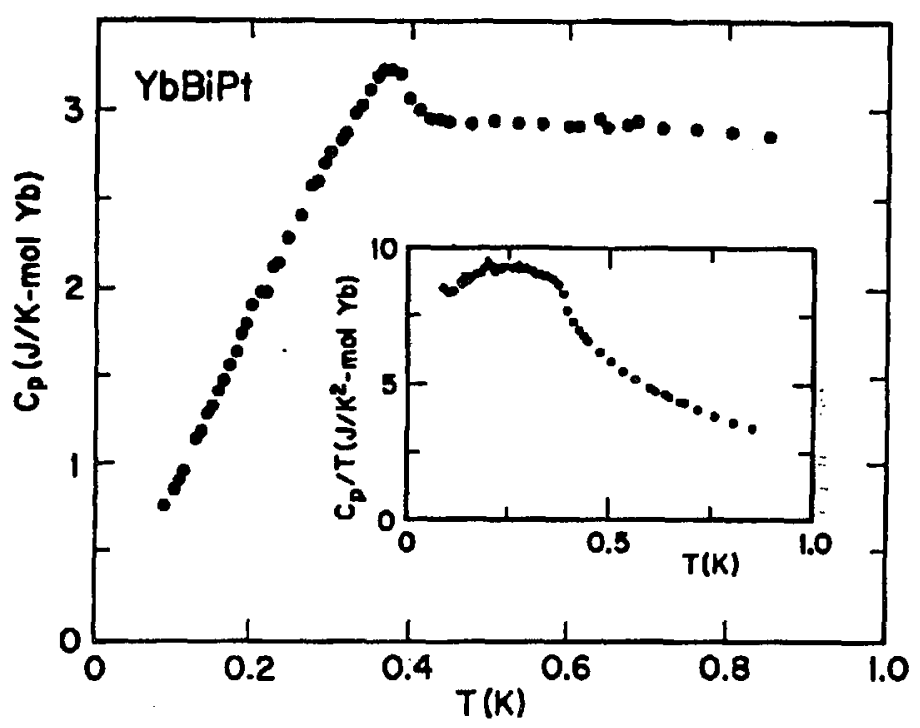

Fig. 3. Specific heat at YbPtBi below $1 \mathrm{~K}$.

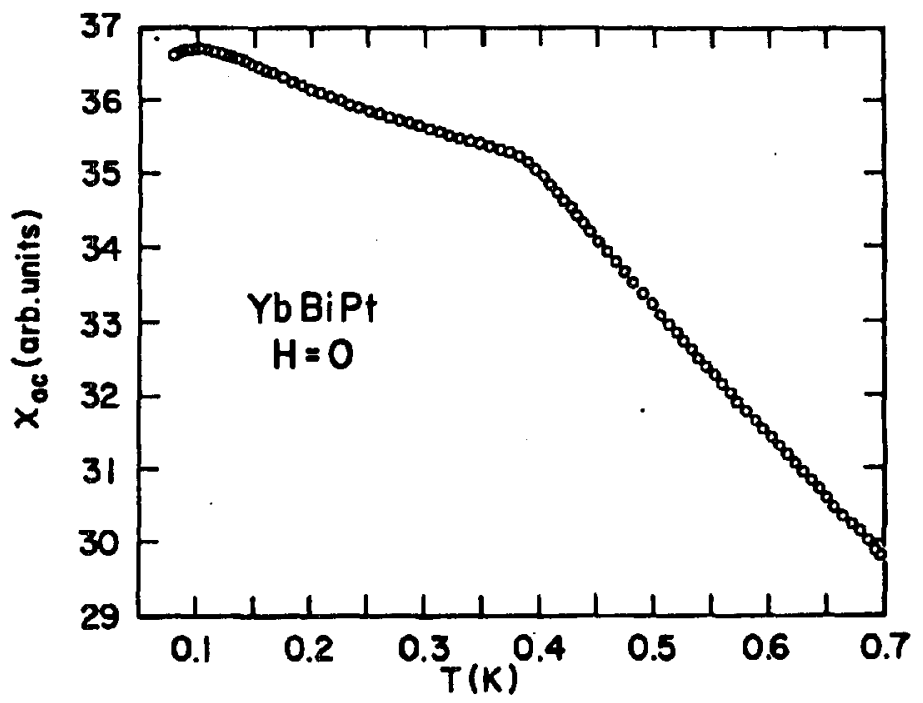

Fig. 4. Low-field ac magnetic susceptibility of YbPtBi below $0.7 \mathrm{~K}$.

for $H=0$ and $T$ between 0.07 and $0.7 \mathrm{~K}$ is shown in Fig. 4 . A distinct kink at $0.39 \mathrm{~K}$ and a rounded maximum at $0.1 \mathrm{~K}$ are the most prominent features. Both these features vary with increasing external magnetic field. The kink is shifted to lower temperatures and is no longer discernible for fields exceeding $2 \mathrm{kOe}$. The rounded maximum occurs at slightly higher temperature at low field values and 
for $H>2 \mathrm{kOe}$ is shifted back again to lower temperature. Varying of the applied frequency $\nu$ of the $\chi_{\text {ac }}$ measurement revealed that the transition is not of spin-glass type because no shift in the kink temperature was observed for $\nu$ varying between a few tens of $\mathrm{s}^{-1}$ and some $\mathrm{kHz}[19]$.

A microscopic study with special attention to this phase transition was attempted with $\mu \mathrm{SR}$ experiments below $1 \mathrm{~K}$ [20]. The zero-field $\mu \mathrm{SR}$ spectra reveal two distinctly different magnetic environments for the $\mu^{+}$decay sites by the observation of two decay channels with distinctly different relaxation rates. As for $\mathrm{CeAl}_{3}$, the percentage of these two magnetically-inequivalent sites changes with temperature whereby the most sizeable variation is ohserved between 0.2 and $0.7 \mathrm{~K}$ (see Fig. 5). From experiments made in external fields it may be concluded, how-

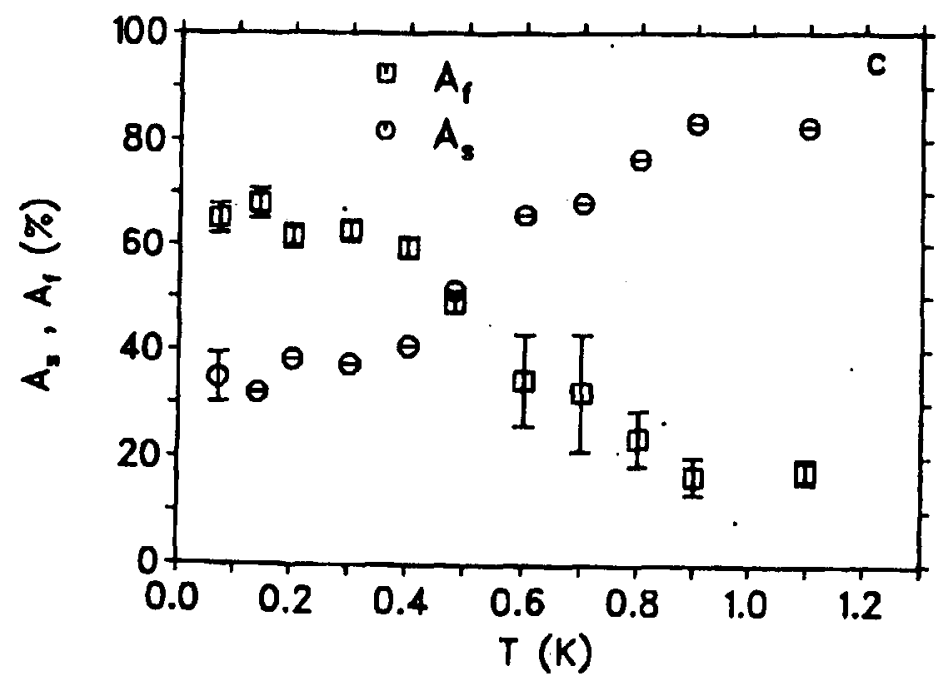

Fig. 5. Temperature dependence of the amplitudes of the slow and fast components of $\mu^{+}$relaxation in YbPtBi below $1.2 \mathrm{~K}$.

ever, that the $\mu^{+}$decay sites are structurally equivalent and therefore again, a magnetic inhomogeneity has to be considered. For $T$ close to $0 \mathrm{~K}$, the magnetically different domains must be distributed with a ratio of about $2: 1$. If at all, the transition manifests itself by an increase in the respective relaxation rates, whereby the increase for the fast-decaying component amounts to a factor of about 6 . Nevertheless, this increase is far from being abrupt in temperature and certainly not comparable to the distinct anomaly in $C_{p}(T)$. Calculations aiming at determining the value of $\mathrm{Yb}$ moments that might explain the $\mu \mathrm{SR}$ results give $0.1 \mu_{\mathrm{B}} / \mathrm{Yb}$ or less. This again implies a tremendous reduction of any reasonable moment to be expected from the $\mathrm{Yb}^{3+}$ configuration.

Very surprising are the results of $C_{p}(T)$ measurements on material where $\mathrm{Yb}$ is partly replaced by $\mathrm{Y}$, i.e., of the type $\mathrm{Yb}_{1-x} \mathrm{Y}_{x} \mathrm{PtBi}$. It was found that the overall magnitude of $C_{p}$ below $1 \mathrm{~K}$ scales with the content of $\mathrm{Yb}$ ions per formula unit, as may be expected. Quite unexpected, however, is the observation that the 
$C_{p}$-anomaly occurs always at the same temperature for $x$ varying between 0 and 0.5 [18]. This implies that the order parameter involved in the transition must be of rather local character. Structural disorder, which might be expected from the features of the unit cell described above is unlikely the cause for this because the sizeable value for the RRR and the structural equivalence of the $\mu^{+}$trapping sites effectively rule out this explanation. It is to be hoped that further microscopic studies with higher-resolution capabilities would help to solve these puzzles. For theoretical efforts it must be kept in mind that various important interactions (crystal-electric field, RKKY exchange interaction, Kondo's compensation) are of almost equal size and small.

\section{3. $U C u_{5}$}

Experimental studies of the low-temperature properties of this compound gave the first evidence that a heavy-electron state may also form in the environment of a magnetically-ordered matrix [21], a feature that was not anticipated in early models for explaining heavy-electron formation. Below we discuss some experimental results which suggest that the ensemble of itinerant electrons consists of essentially independent subsystems. Various macroscopic and microscopic measurements established the rather conventional antiferromagnetic order among ionic $\mathrm{U}$ moments of respectable value of the order of $1 \mu_{\mathrm{B}} / \mathrm{U}$ [22-24]. The corresponding phase transition occurs at $15 \mathrm{~K}$. Clear manifestations of this transition are anomalies in the temperature dependence of the specific heat $C_{p}(T)$ and the electrical resistivity $\rho(T)$. The feature of the latter anomaly implies that the transition induces a partial gapping at the Fermi surface thereby reducing the amount of a vailable itinerant charge carriers [25]. The temperature dependence of $\rho$ between 0.02 and $30 \mathrm{~K}$ is shown in Fig. 6 . It reveals that the remaining free carriers experience a drastic reduction in scattering below about $12 \mathrm{~K}$, a combined effect of reduced magnetic scattering and the gradual onset of coherence with decreasing temperature.

This coherence leads to a distinct increase in the $C_{p} / T$-ratio with decreasing temperature below $4 \mathrm{~K}$, rising to a value exceeding $300 \mathrm{~mJ} /\left(\right.$ mole $\left.\mathrm{K}^{2}\right)$ below $2 \mathrm{~K}$. This correlated electronic subsystem now, by itself, loses its stability and another phase transition at approximately $1 \mathrm{~K}$ is indicated again by anomalies of the specific heat and $\rho(T)$ (see Fig. 6). Below $1 \mathrm{~K}, \rho(T)$ reaches a maximum at $0.4 \mathrm{~K}$ and subsequently decreases somewhat as $T$ approaches zero kelvin. The anomaly of $C_{p}(T)$ is distinct but small, only little entropy change is involved. Below $0.7 \mathrm{~K}, C_{p}$ decreases linearly in temperature to zero, i.e., $C_{p}=\gamma T$, whereby $\gamma=$ $80 \mathrm{~mJ} /\left(\right.$ mole K$\left.^{2}\right)$ [21]. These two observations again imply a substantial reduction of the Fermi surface and in combination with additional transport and spectroscopic measurements it may be concluded that the now remaining itinerant electrons form a state with the features of a renormalized Fermi liquid as $T \rightarrow 0 \mathrm{~K}$. This state, however, coexists with a conventional magnetically-ordered state, certainly stabilized by RKKY interactions, and yet another ordered phase, developing below $1 \mathrm{~K}$, whose order parameter is not yet established.

Rather unexpectedly this $1 \mathrm{~K}$ transition does not influence in any detectable 


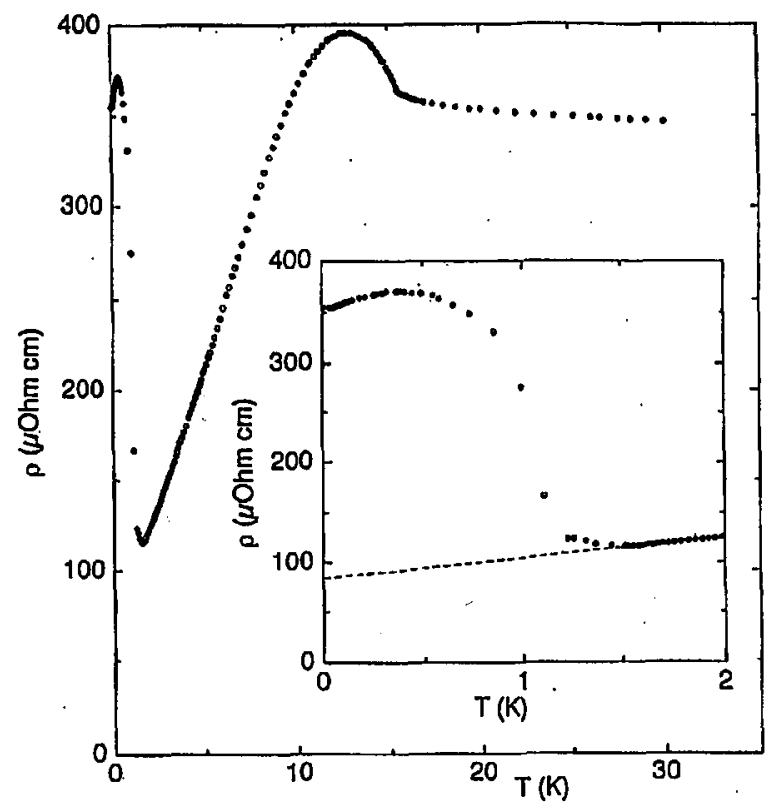

Fig. 6. Temperature dependence of the electrical resistivity of $\mathrm{UCu}_{5}$ below $30 \mathrm{~K}$. The inset shows the data obtained below $2 \mathrm{~K}$ on an expanded temperature scale.

way the magnetically-ordered state that develops below $15 \mathrm{~K}$. This became obvious from recent $\mu \mathrm{SR}$ - and neutron experiments probing the magnetism of $\mathrm{UCu}_{5}$ below $15 \mathrm{~K}$ [24]. Within experimental resolution, no alteration in either the magnetic structure or the magnitude of the ordered moments at $1 \mathrm{~K}$ could be observed. The only microscopic manifestation of this transition in $\mu \mathrm{SR}$ or neutron scattering is an increase in the relaxation rate in the $\mu^{+}$-decay spectra. This is shown for one relaxation channel in Fig. 7. A possible implication of this result may be the formation of order among tiny moments that would escape the detection with neutron techniques but drastically influence the heavy-electron state that develops below $4 \mathrm{~K}$. Estimating the possible magnitude of moments involved in the transition from $\mu \mathrm{SR}$ data results in values of $0.1 \mu_{\mathrm{B}}$ or less. Another conjecture, the formation of a small internal distortion of the crystal lattice, i.e., the formation of a charge-density wave does not seem to be compatible with the combined $\mu \mathrm{SR}$ and neutron results.

The $1 \mathrm{~K}$ transition is extremely sensitive to impurities and imperfections in general, as is often observed for heavy-electron systems. For some deliberately introduced impurities, especially those on Cu sites, this is also true for the $15 \mathrm{~K}$ transition, in not the same excessive way, however [25]. The conjecture of the coexistence of two essentially decoupled electronic subsystems found some support in recent thermal expansion measurements [26].

The major question of how this separation within the subsystem of the conduction electrons might occur is still a puzzle. Estimates based on band-structure 


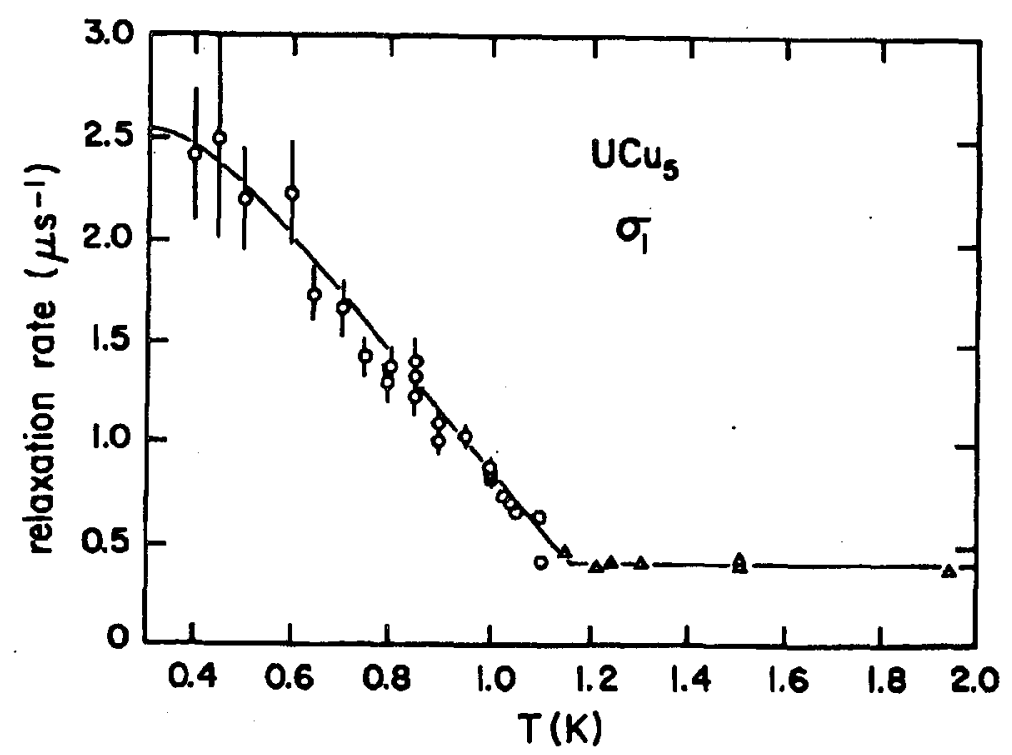

Fig. 7. Temperature dependence of the $\mu \mathrm{SR}$ relaxation rate for one decay channel in $\mathrm{UCu}_{5}$ below $2 \mathrm{~K}$.

calculations reproduce both the Néel temperature and the magnitude of the order moment of the conventional antiferromagnetic order quite well [27]. There is clearly no hint, however, of the behaviour at lower temperatures described above. Considering the crystal structure of $\mathrm{UCu}_{5}$ it may be noted that cation- and anion sites are structurally well separated and this may lead to an intrinsic anisotropy of possible interactions. Nevertheless, the fact that a heavy-electron state may also form even when the $f$-electron moments are aligned in a conventional magnetic order seems of some significance for theoretical models describing corresponding correlated systems.

$$
\text { 2.4. } U_{1-x} T h_{x} B e_{13-y} B_{y}
$$

Superconductivity in this type of compounds occurs out a highly resistive and strongly paramagnetic normal state [28]. Various experimental results indicate that the superconducting state in pure $\mathrm{UBe}_{13}$ is unconventional and characterized by gap zeros (see e.g. [29]). Magnetic and non-magnetic substitutions on either the $\mathrm{U}$ or the Be sites usually lower the critical temperature $T_{\mathrm{c}}$ for superconductivity very effectively and result in extensions of the gap zeros over appreciable parts of the Fermi surface already for small concentrations of impurity elements. Outstanding exceptions are replacements of $\mathrm{U}$ by $\mathrm{Th}$ and $\mathrm{Be}$ by $\mathrm{B}$ on a low atomic-percent level. Experimental observations concerning the properties of correspondingly manipulated materials have been published before [30, 31].

The most amazing results of Th substitutions of concentrations $x$ is the non-monotonic dependence of $T_{\mathrm{c}}(x)$ and the appearance of a second phase transition in the superconducting state which is, however, restricted to the concentration 
range $0.02<x<0.045$. The corresponding phase boundaries combine to a highly unusual superconducting phase diagram [32] and it has been conjectured that superconducting phases with different types of order parameters are realized in this compound series. This phase diagram, as it follows from measurements of the low-temperature specific heat $C_{p}(T)$ is shown in Fig. 8. Microscopic measurements

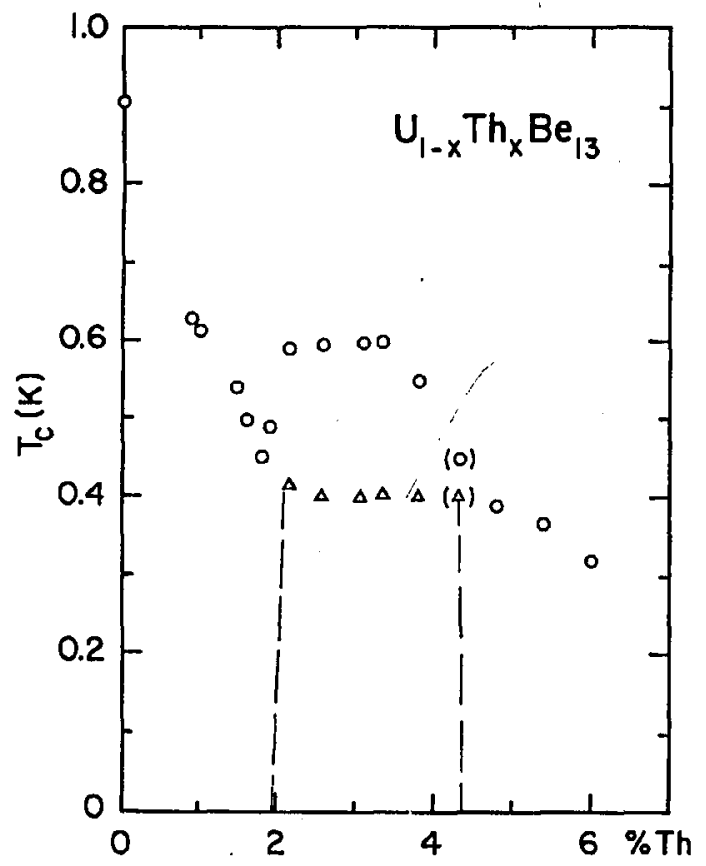

Fig. 8. Superconducting phase diagram of $\mathrm{U}_{1-x} \mathrm{Th}_{x} \mathrm{Be}_{13}$.

using the $\mu S R$ technique [33] indicate that in the superconducting phase denoted as $L$, superconductivity either coexists with ordered magnetic moments of the order of $10^{-2} \mu_{\mathrm{B}} / \mathrm{U}$ or that the superconducting order parameter carriers a magnetic component. Model calculations based on a Ginzburg-Landau-type analysis [34] indicate that the second possibility may indeed be realized. The first possibility would again require the coexistence of two long-range order phenomena, in this case of superconductivity with some small-moment magnetic order, both involving the heavy quasiparticles.

Boron doping on the Be sites in relatively small quantities has a drastic effect on the $C_{p}$ anomaly at the superconducting transition [31,35]. This is exemplified in Fig. 9, where it may be seen that for $y \approx 0.03$, the discontinuity $\Delta C_{p}$ at $T_{c}$ is about twice as large as that expected from the weak-coupling BCS calculation and we also note that the shape of the anomaly is distinctly different from the usual mean-field behaviour and shows a tendency towards a $\lambda$-type anomaly. The reason for this drastic change in spite of only a small alteration of the chemical composition of the material is not known. One possibility is a drastic reduction of 
the superconducting coherence length upon doping with $\mathrm{B}$ but it is again not clear how this should happen by the addition of only a small percentage of $B$ atoms per formula unit.

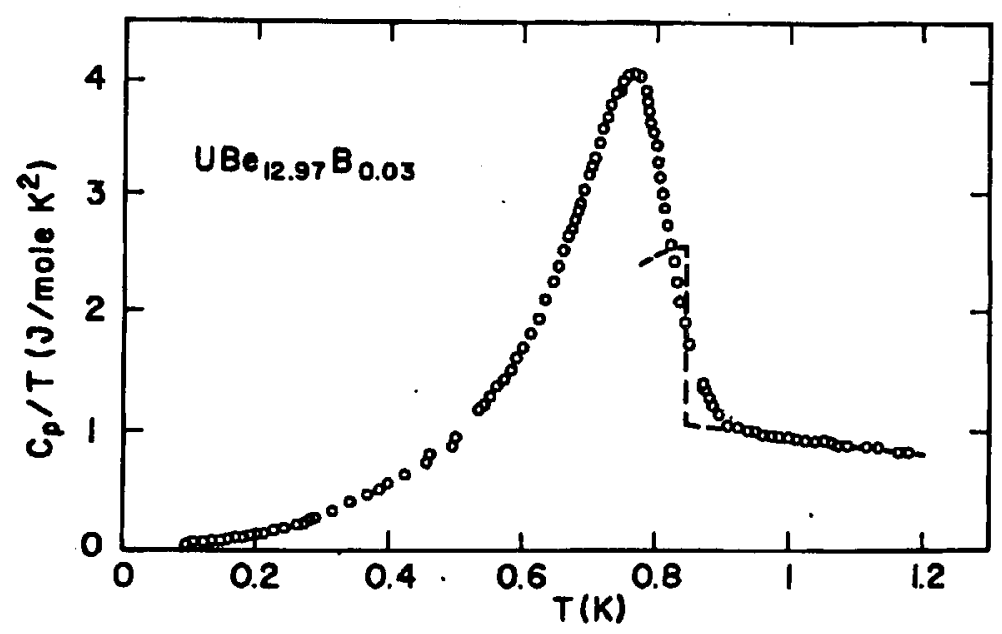

Fig. 9. $C_{p} / T$ ratio of Boron-doped $\mathrm{UBe}_{13}$ below $1.2 \mathrm{~K}$. The broken line indicates the expected BCS anomaly in the weak-coupling limit.

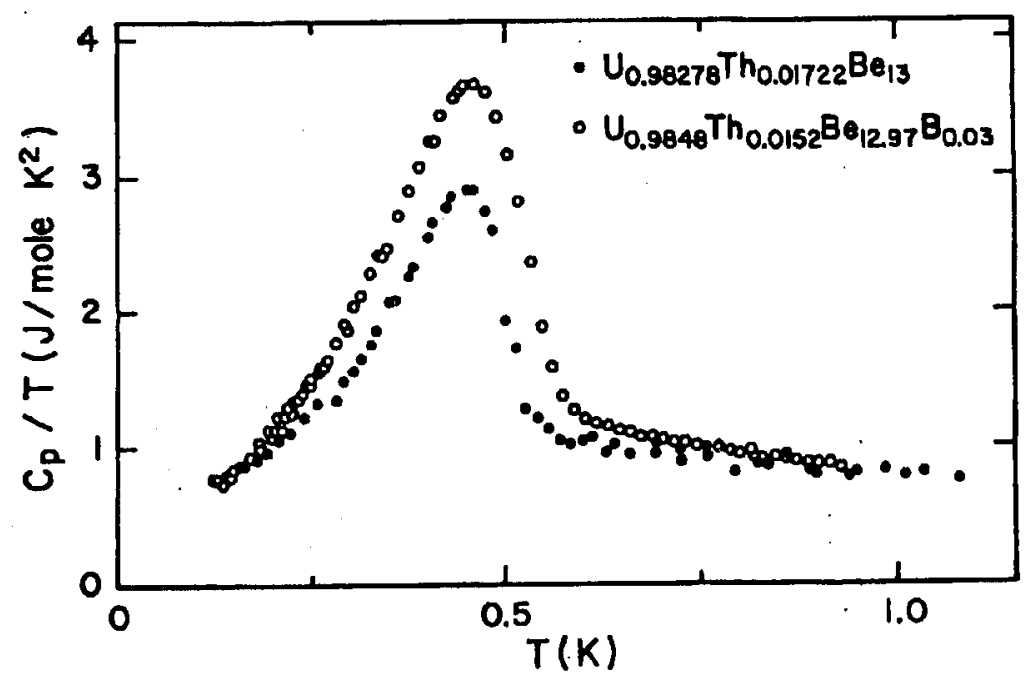

Fig. 10. $C_{p} / T$ ratio of superconducting $\mathrm{U}_{1-x} \mathrm{Th}_{x} \mathrm{Be}_{13-y} \mathrm{~B}_{y}$ compounds below $1 \mathrm{~K}$.

A similar anomaly enhancement is observed for Th-doped $\mathrm{UBe}_{13}$. This is demonstrated for one selected example in Fig. 10, where the respective ratios $C_{p} / T$ are plotted versus temperature. This figure emphasizes, however, yet another 
problem that arises if one considers the requirement of entropy balance that should be obeyed in this type of phase transition. It is quite obvious that this requirement cannot be met without assuming that the hypothetical normal state of these materials carries some hidden degrees of freedom at temperatures below $T_{\mathrm{c}}$. The corresponding specific heat, assumed to be described by $C_{p}=\gamma(T) \cdot T$, would necessarily result in a $\gamma$-value at zero temperature of the order of $\gamma(0)=3 \mathrm{~J} /\left(\right.$ mole K$\left.^{2}\right)$. Again it is not clear where these degrees of freedom originate. Suppressing the superconducting state with large external magnetic fields and probing $C_{p}(t)$ under these conditions is not an advisable way to solve this puzzle, because magnetic fields of the required magnitude certainly influence the electronic characteristics of the normal state that would be established in zero external field at temperatures below $T_{\mathrm{c}}$ and therefore any conclusion drawn from such an experiment is necessarily ambiguous.

\section{Conclusion}

The above presented, especially selected examples are intended to demonstrate that ground-state properties of materials termed as heavy-electron systems are not really well understood. It appears that at least in some cases, the experimentally established properties are not easily reconciled with expectations of behaviour that follow from models assuming a simple Fermi-liquid to be the general ground state of these systems.

\section{Acknowledgments}

All of the experimental data presented and discussed here were obtained in collaborations with many colleagues, lasting many years. I am very grateful to all of them for sharing their working power and enthusiasm over an extended period of time. In particular I am indebted to E. Felder, A. Bernasconi, J. Gavilano, A. Schenck, Z. Fisk and R.H. Heffner. Much of this work was supported financially by the Schweizerische Nationalfonds zur Förderung der wissenschaftlichen Forschung.

\section{References}

[1] J. Friedel, Can. J. Phys. 34, 1190 (1956).

[2] A. Blandin, J. Friedel, J. Phys. Radium 19, 573 (1958).

[3] P.W. Anderson, Phys. Rev. 124, 41 (1961).

[4] J. Kondo, Prog. Theor. Phys. 32, 37 (1964).

[5] T. Kasuya, Prog. Theor. Phys. 16, 45 (1956).

[6] K. Yosida, Phys. Rev. 106, 893 (1957).

[7] B.A. Jones, Physica B 171, 53 (1991).

[8] K. Andres, J.E. Graebner, H.R. Ott, Phys. Rev. Lett. 35, 1979 (1975).

[9] H.R. Ott, O. Marti, F. Hulliger, Solid State Commun. 49, 1129 (1984).

[10] S. Barth, H.R. Ott, F.N. Gygax, B. Hitti, E. Lippelt, A. Schenck, C. Baines, V. van den Brandt, T. Konter, S. Mango, Phys. Rev. Lett. 59, 2991 (1987).

[11] S. Barth, H.R. Ott, F.N. Gygax, B. Hitti, E. Lippelt, A. Schenck, C. Baines, Phys. Rev. B 39, 11695 (1989). 
[12] A. Schenck, in: Frontiers in Solid State Sciences, vol. on Magnetism, Eds. L.C. Gupta, M.S. Multani, World Scientific, Singapore, in print.

[13] J. Hunziker, J.L. Gavilano, S. Büchi, H.R. Ott, Physica B, in print.

[14] J.L. Gavilano, J. Hunziker, H.R. Ott, Physica B, in print.

[15] P.C. Canfield, J.D. Thompson, W.P. Beyermann, A. Lacerda, M.F. Hundley, E. Peterson, Z. Fisk, H.R. Ott, J. Appl. Phys. 70, 5800 (1991).

[16] P.C. Canfield, private communication.

[17] Z. Fisk, P.C. Canfield, W.P. Beyermann, J.D. Thompson, M.F. Hundley, H.R. Ott, E. Felder, M.B. Maple, M.A. Lopez de la Torre, P. Visani, C.L. Seaman, Phys. Rev. Lett. 67, 3310 (1991).

[18] J.P. Thompson, P.C. Canfield, A. Lacerda, M.F. Hundley, Z. Fisk, H.R. Ott, E. Felder, M. Chernikov, M.B. Maple, P. Visani, C.L. Seaman, M.A. Lopez de la Torre, G. Aeppli, Physica B 186-188, 355 (1993).

[19] M. Chernikov, H.R. Ott, P.C. Canfield, Z. Fisk, unpublished.

[20] A. Amato, P.C. Canfield, R. Feyerherm, Z. Fisk. F.N. Gygax, R.H. Heffner, D.E. MacLaughlin, H.R. Ott, A. Schenck, J.D. Thompson, Phys. Rev. B 46, 3151 (1992).

[21] H.R. Ott, H. Rudigier, E. Felder, Z. Fisk, B. Batlogg, Phys. Rev. Lett. 55, 1595 (1985).

[22] H.J. van Daal, K.H.J. Buschow, P.B. van Aken, M.H. van Maaren, Phys. Rev. Lett. 34, 1457 (1975).

[23] A. Murasik, S. Ligenza, A Zygmunt, Phys. Status Solidi A 23, K163 (1974).

[24] A. Schenck, P. Birrer, F.N. Gygax, B. Hitti, E. Lippelt, M. Weber, P. Böni, P. Fischer, H.R. Ott, Z. Fisk, Phys. Rev. Lett. 65, 2454 (1990).

[25] H.R. Ott, Z. Fisk, in: The Challenge of $d$ and $f$ Electrons, ACS Symposium Series 394, Eds. P.R. Salahub, M.C. Zerner, Am. Chem. Soc., Washington 1989, p. 260.

[26] J. Clayhold, C. Reyl; H.R. Ott, unpublished.

[27] M.R. Norman, B.I. Min, T. Oguchi, A.J. Freeman, Phys. Rev. B 38, 6818 (1988).

[28].H.R. Ott, H. Rudigier, Z. Fisk, J.L. Simith, Phys. Rev. Lett. 50, 1595 (1983).

[29] Z. Fisk, H.R. Ott; Int. J. Mod. Phys. B 3, 535 (1989).

[30] H.R. Ott, H. Rudigier, Z. Fisk, J.L. Smith, Phys. Rev. B 33, 126 (1986).

[31] H.R. Ott, E. Felder, Z. Fisk, R.H. Heffner, J.L. Smith, Phys. Rev. B 44, 7081 (1991).

[32] H.R. Ott, J. Magn. Magn. Mater. 108, 1 (1992).

[33] R.H. Heffner, J.L. Smith, J.O. Willis, P. Birrer, C. Baines, F.N. Gygax, B. Hitti, E. Lippelt, H.R. Ott, A. Schenck, E.A. Knetsch, J.A. Mydosh, D.E. MacLaughlin, Phys. Rev. Lett. 65, 2816 (1990).

[34] M. Sigrist, T.M. Rice, Phys. Rev. B 39, 2200 (1989).

[35] Z. Fisk, H. Borges, M. McElfresh, J.L. Smith, J.D. Thompson, H.R. Ott, G. Aeppli, E. Buchner, S.E. Lambert, M.B. Maple, C. Broholm, J.K. Kjems, Physica C 153-155, 1728 (1987). 